\title{
Optimization of Transit Ridership Using Parallel Negative Algorithm
}

\author{
Caixia Han \\ School of Information technology, College of science \&Arts of Jianghan University, China
}

Keywords: Macroscopic models, transit vehicles, optimizer.

\begin{abstract}
Macroscopic models are in general not suit-able for modeling adaptive ridership control since they do not consider individual vehicle arrivals, which are a necessary input to adaptive traffic ridership. The use of a global weighting factor also unavoidably assumes that the passengers in a transit vehicle enter an intersection one at a time, rather than all at once, thus tends to overstate the impact of transit vehicles. Although microscopic simulation models based on vehicle dynamics have the ability to track the behavior and status of individual vehicles, the computation required to track, the simulation results show that the PGA-based optimizer for adaptive TRP outperformed the fully actuated NEMA control in all test cases. The results also show that the PGA-based optimizer can produce TSP timing plans that benefit the transit vehicles while minimizing the impact of TSP on the general vehicles.
\end{abstract}

\section{Introduction}

Transit ridership priority (TRP) is designed to help transit vehicles to cross ridershipized intersections with less delay by modifying the normal ridership operations. TSP is increasingly becoming a dominant form of preferential treatment for transit vehicles along urban arterials. There are three categories of TRP priority strategies: passive, active, and adaptive. Passive priority operates continuously regardless of whether a transit vehicle is present, while active (actuated) priority is responsive to the requests of transit service based on a predetermined ridership priority design. The state-of-the-art studies on TSP are predominantly focused on adaptive strategies, which are not only responsive to transit requests in real-time, but also to current traffic conditions through real-time optimization of select performance criteria such as vehicle delay and stops.

\section{Accessibility Function}

Accessibility is considered as one of the most important factors affecting public transit ridership. Among all the functions used to identify accessibility, the two most representative functions are the Negative Exponential Function and Negative Logistic Function. Based on a 126,000 sample size analysis of city Portvehicle, Kimpel et al. concluded that the Negative Logistic Function adopts passenger flow better than the Negative Exponential Function .

The relationship between the probability of public transit trip production and the distance between origin and a transit site can be expressed using the Negative Logistic Function as follows:

$A D(i, j, k, t)=\frac{1}{k} \sum_{i} \sum_{j} \sum_{k}\left\{[1+w(t)]\left[\left(D_{j, k}^{i}(t)-A_{j, k}^{i}(t)\right)\right]\right\}$

Where:

A represents the probability railway intersection trip production;

D represents the distance from origin to a railway intersection site;

$\mathrm{A}$ and $\mathrm{D}$ are parameters.

In equation (1), values of a and $d$ indicate the extent of demand for travel with distance decay. Figure 1 shows several combinations of curves. Most passengers walk to transit within the distance of 400-800 meters. Taking into account the non-linear coefficient of the road network, we define a 600 meters buffer zone from a transit site as the affected area. To facilitate the presentation, we use M207 to represent the 600 meters affected area, while the value of a is 2.0 and the value of $b$ is 0.007 . 


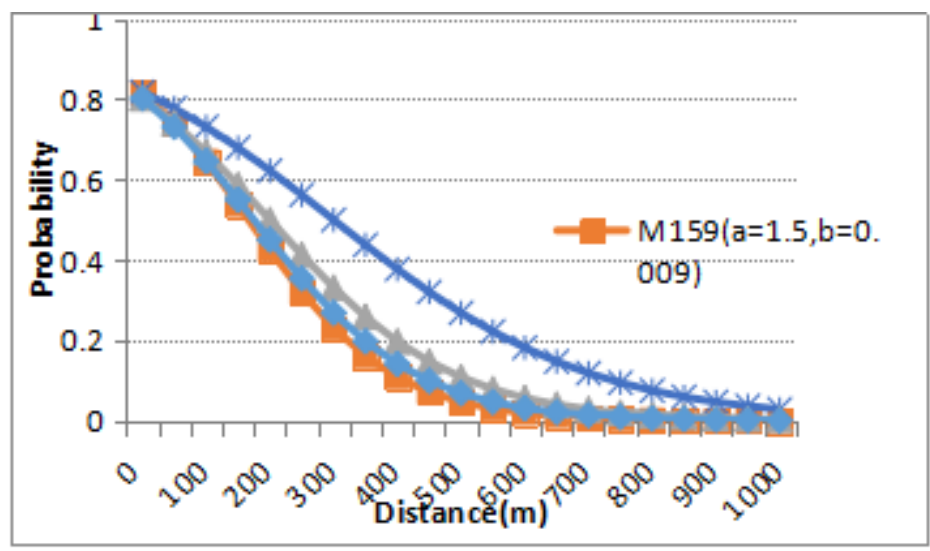

Fig. 1 Curves of negative logistic model under different parameter sets

\section{Spatial Decomposition of Population}

In order to predict the demand of public transit using accessibility model, population distribution data is required. Because grid cell units can better respond to the relationship between data and space, we use data decomposition to break down large statistical units, such as total number of population, into small grid cells to avoid illusion caused by the expression of population density and the mean value on different administrative units [2].

In a general grid-based data model, a large statistical unit (subdivision or streets) is broken into small grid cells. A more intuitive method is to allocate data onto each grid based on the type of vehicle use and development intensity. However, it takes an extremely detailed field survey and massive amount of work to determine the development intensity on each grid. An effective way to solve this problem is to use a random allocation algorithm. Wegener used Monte Carlo simulation to disaggregate household and employment data to $30 \mathrm{~m}$ grid to simulate vehicle use patterns, transportation activities, and their impacts [3]. Due to the complexity of vehicle use in most Chinese cities, Huang et al. introduced a doubly weighted approach into the Monte Carlo algorithm to obtain more accurate distribution of micro-level social and economic activities. On the basis of vehicle-use zoning restrictions, this method divides vehicle into homogeneous regions where development intensity is close. Under the dual constraints of vehicle use type and homogeneous area, Monte Carlo simulation is carried out for data disaggregation. The case study of Wuhan, China shows that microscopic analysis is more suitable for vehicle use and transport activities than using large spatial units [4].

\section{Case Study of Wuhan}

Based on the above prediction method, model parameters need to be addressed in the accessibility model to forecast transit site passenger flow. We use Wuhan City Rail Line 1 for example to test the parameters and verify the feasibility of the method.

Wuhan is the capital of Hubei Province, one of China's major urban centers. There are currently three railway intersection lines, line 1, line 2, and line 4, respectively opened in July 2004, December 2012, and December 2013. Since line 2 and line 4 have opened only for a short time, the daily ridership is not traffic is not yet stable. This study only analyzed traffic data for line 1. 


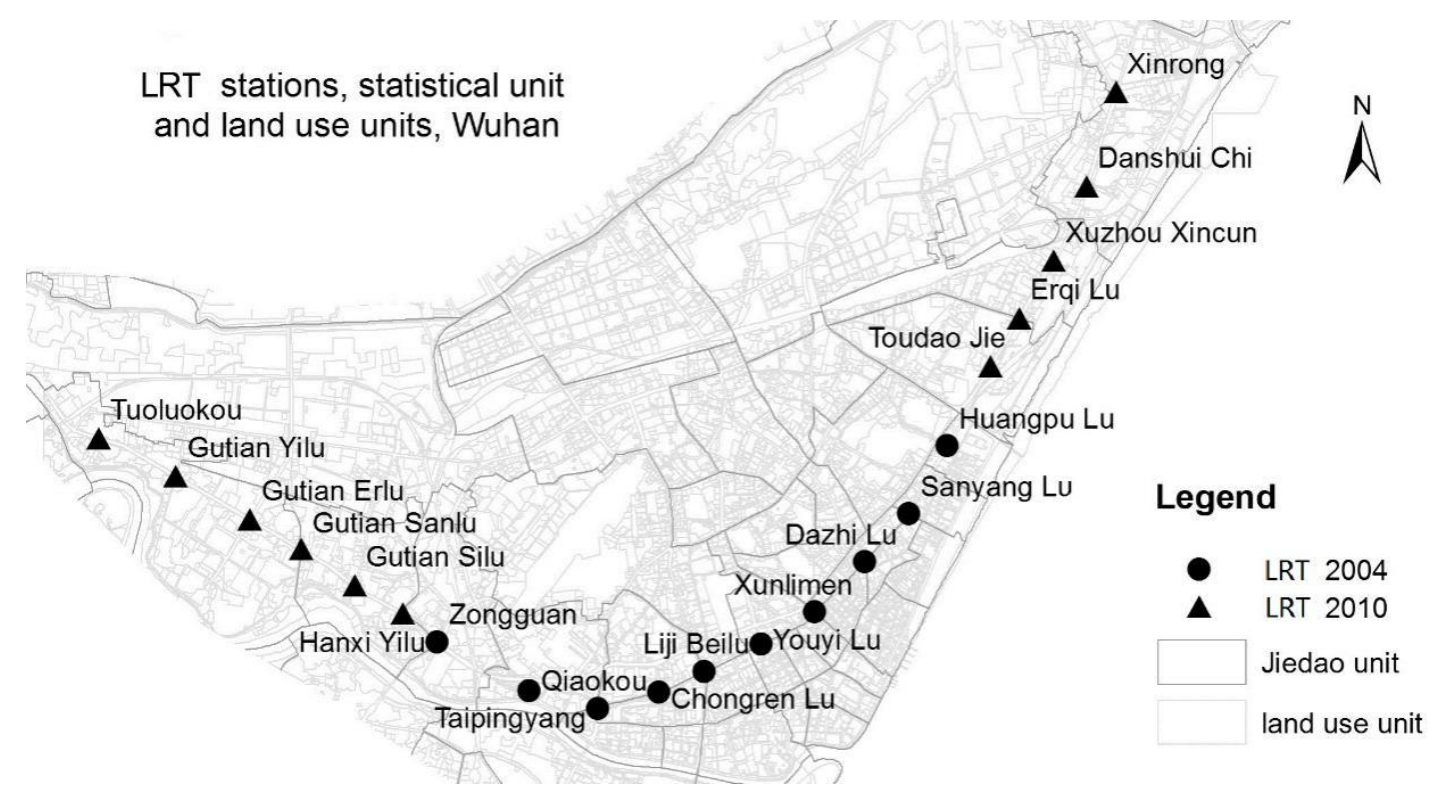

Fig 2 Stations of Wuhan Rail Line 1

Due to the availability of data, we select one week in March 2011 to analyze the weekday ridership. Morning peak hour passenger flow is shown in Figure 2. First, it indicates more concentrated segments of passengers between Chongren Lu station and Huangpu Lu station. This area is a central CBD area with high vehicle development intensity and high density of road network. Secondly, there is a large number of new residential subdivisions between Gutian Yilu station and Zongguan Station with more balanced vehicle development intensity. The Taipingyang station is located close to the Han River where vehicle use intensity is low. Therefore, the passenger flow is relatively lower at this station.

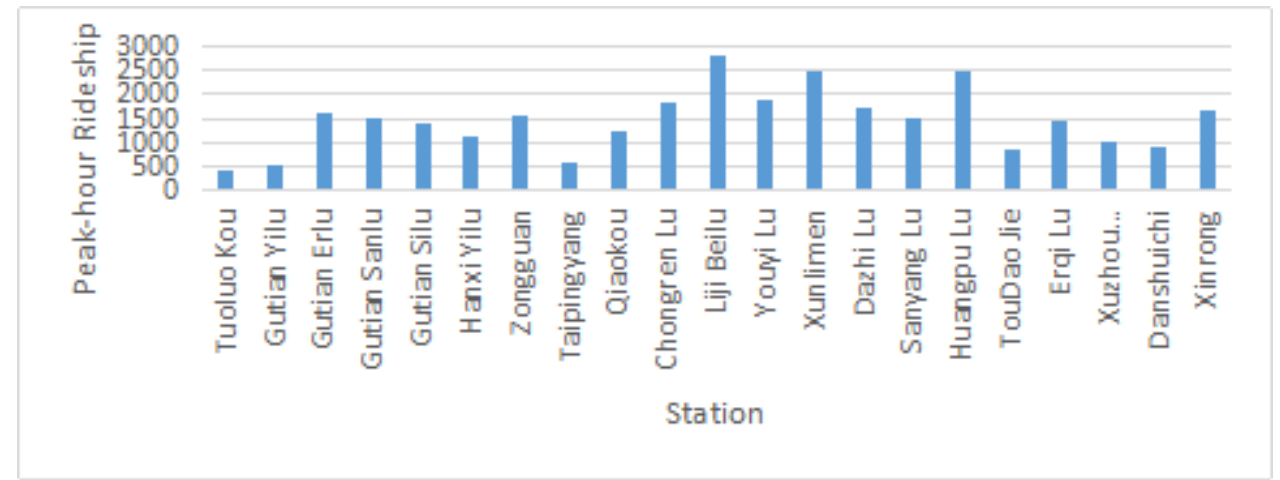

Fig 3.Morning peak-hour ridership on stations of Wuhan Rail Line 1

In this study, a size $30 \times 30$ grid population distribution is obtained by using 2010 census population data, 2010 Wuhan vehicle use data, and Doubly Weight Monte Carlo approach.

\section{Analysis and Discussion}

As shown above, when parameters $\mathrm{a}$ and $\mathrm{b}$ are valued properly, this method produces accurate prediction results. There are many factors affect passenger flow, such as ticket price, level of income, degree of transit network coverage, the ease of transfer, bus routes connections, and etc. Site accessibility based passenger flow prediction mainly considers the vehicle use type around a transit site, but ignoring many other affecting variables. The accuracy of results can be impaired. However, fewer amount of analytical data is required using this prediction method. Another advantage is that this prediction method has low cost on model construction in a short period of time. Considering the large scale development and rapid constructions in most Chinese cities, this method adapts different situations quickly while for further study to improve its prediction results. 


\section{Determination of Parameters}

There are two key factors, parameters a and b, affecting prediction results in the accessibility function. Because the concurrent actual population data and railway intersection ridership data in each divided grid cell are unavailable, the only alternative is to use fitting method to determine the values of parameters. Along with the completeness of survey data, for example the number of residents and the number of railway intersection users from each residential building, accurate values of $\mathrm{a}$ and $\mathrm{b}$ can be obtained by computing the corresponding relationship between $\mathrm{p}$ and $\mathrm{d}$.

Analyzing Figure 3, it can be found that the most suitable values of $a$ and $b$ are different on different sites., b different values. The M155 is fit for Gutiansanlu station, Gutiansilu station, Zongguan station, Xuzhouxingchun station. The proportion of residential vehicle nearby those sites is from $50 \%$ to $67 \%$. Most of the vehicle is new residential with balanced development intensity. The M147 is fit Hanxiyilu station, Qiaokoulu station, Chongrenlu station, Youyilu station. The proportion of residential vehicle surrounding them is from $62 \%$ to $75 \%$. Both the new neighborhood with high intensity and old neighborhood with low intensity are around the site. It is visible that different degree of mixing and development intensity of vehicle use around the site, the appropriate of $a$ and $b$ parameters are different. The different types of sites have different values of $a$ and $b$.

\section{Distance Computing and Service Area Division}

The distance for passenger to walk from origin to a railway intersection site should be the actual walking length. Currently, it is calculated using the value $d$, which represents a linear distance between a grid cell and a site.

Using GIS, we can easily get the service area for each site, and assigned kinds of spatial data to each site. However, the passengers who are within the overlapping region will face multiple options. The simplest way is to rank the travel to the nearest site using Thiessen Polygons method. A further approach is to build an accurate walking road network to calculate the precise walking distance, and simulate the probability of travelers with random selection function [5].

\section{Conclusion}

According to the survey data in Wuhan, about $70 \%$ of the total railway intersection riders walk to the transit sites, and proximally $14 \%$ of the rest use bus transit to reach the railway intersection sites. railway intersection ridership is affected by the bus route network coverage, distance from bus stop to transit site, and travel behaviors. To obtain accurate predicting results, many factors need to be carefully examined with large quantity of data. The direct passenger flow prediction method indicates that, after verifying the relevance between the total number of daily bus routes and total number of passengers, the passenger flow from each bus route can be estimated based on the bus routes coverage and distance to the railway intersection sites. The prediction results can be improved when data demand is neutral.

\section{References}

[1] R.Untermann, Accommodating the pedestrian: Adapting towns and neighbourhoods for walking and bicycling.[D] Van Nostrand Reinhold, New York, 1984.

[2] Cervero R. Rail-oriented office development in California: how successful? [J].Transportation Quarterly, 1994,48(1):33-44

[3] HUANG S, GUAN H Z, YAN H, A study on urban railway connection mode selections in Beijing[J]. China Civil Engineering Journal, 2009, 42(7): 126-130

[4] GUO T. Research on TOD Reasonable Area around Urban Rail Traffic Site-A Case Study of West Jiangnan Station Guangzhou No2 Subway[J]. Planners, 2008, 24(3): 75-78

[5] Levinson H S, O Brown-West. Estimating bus ridership[J].Transportation Research Record, 1984,994:8-12 